\section{An ideal signal generator for time-sampling observation procedures}

\section{JAMES H. REYNIERSE and JAMES W. TOEVS Hope College, Holland, Michigan 49423}

Systematic observation of behavior has been used in a variety of naturalistic and laboratory situations by both ethologists and psychologists. Many of the recording techniques used in these situations involve time-sampling procedures in which observed and recorded events are sampled during specified time units. Such an approach is particularly appropriate when frequency measures, rather than response duration, behavior sequences, etc. are of primary interest. Time sampling can take several forms, but in each case preselected behavioral categories are recorded at regular time intervals. These time intervals are usually signaled by an external stimulus, such as the click of a metronome.

Although widely used, such procedures have a number of limiting deficiencies. Most profoundly, the presence of an external signal not only defines time units for the $O$, but also serve as an extraneous source of stimulation for the observed Ss. Such sources of potential distraction may distort environmental conditions in important unknown ways. In addition, devices such as metronomes have only a limited short range of time intervals, while some time-sampling procedures require relatively large time intervals. Then, too, available devices are often large or require an external power source, making it awkward or even impossible to use in field investigations.

The present paper describes an inexpensive signal generator which has none of these deficiencies. It is a small compact instrument, which can fit comfortably into a shirt or jacket pocket and be taken anywhere, is battery operated, is infinitely variable from about 5 pulses/sec to at least $30 \mathrm{sec}$, and delivers a tone signal only to the $\mathrm{O}$. As such, it is ideally suited for both laboratory and field investigations which require time-sampling observation procedures.

The device is constructed around two Signetics
NE555V timer integrated circuits (Fig. 1). The first timer circuit is wired as a free-running asymmetric astable multivibrator. A logic state 0 output from this circuit (Terminal 3) gates the second timer circuit by providing its ground reference (Terminal 1). The second timer circuit is a symmetric astable multivibrator which oscillates at approximately $1,000 \mathrm{~Hz}$ for the duration of the logic 0 output from the first timer. The $4.7 \mathrm{~K}$ resistor between Terminals 6 and 7 of the first timer and the $30-\mu \mathrm{F}$ capacitor set this duration to about $0.1 \mathrm{sec}$. The interval between pulses (Logic State 1 output) is determined by the $30-\mu \mathrm{F}$ capacitor and the adjustment of the $1 \mathrm{M}$ potentiometer. The NE555V circuit can source or sink up to $200 \mathrm{~mA}$, so it is necessary to place a resistor, $\mathbf{R}_{s}$, in series with the speaker to limit the load current. A value of $1 \mathrm{~K}$ proved optimum for the small dynamic earphones used in this device, however, a smaller value of $R_{s}$ would permit the use of a regular loudspeaker for lecture demonstrations, etc.

Two elements of this diagram are particularly important and require special attention. First, the duration of the tone pulse signal is important for some time-sampling studies, since if a pulse is too long a decision rule must be established for making observation judgments at regular points, such as signal onset, the middle of the signal, at signal offset, etc. While such rules are arbitrary, they can be dispensed with entirely if the pulse signal is clearly momentary without any perceived duration. If the pulse is too long, the $4.7 \mathrm{~K}$ resistor between Terminals 6 and 7 can be replaced with, e.g., a $2.2 \mathrm{~K}$ resistor, which would shorten the pulse even further. Second, the range of potential time intervals can be increased by using a potentiometer of higher resistance than 1 megohm. On the other hand, changing to a lower resistance would yield better control, i.e., resolution, for short intervals.

The individual components for each unit include: one $15 \mathrm{~K}$ resistor $(1 / 4$ or $1 / 2 \mathrm{~W})$, two $4.7 \mathrm{~K}$ resistors $(1 / 4$ or $1 / 2 \mathrm{~W})$, two $1 \mathrm{~K}$ resistors $(1 / 4$ or $1 / 2 \mathrm{~W})$, one $30-\mu \mathrm{F} / 10 \mathrm{~V}$ capacitor, one $.1-\mu \mathrm{F}$ capacitor, two $.01-\mu \mathrm{F}$ capacitors, one 1-megohm potentiometer, two Signetics NE555V linear integrated circuit timers, one dynamic earphone plug and miniature phone jack, one $9 . \mathrm{V}$ battery and battery
Fig. 1. The circuit diagram for signal generator units.

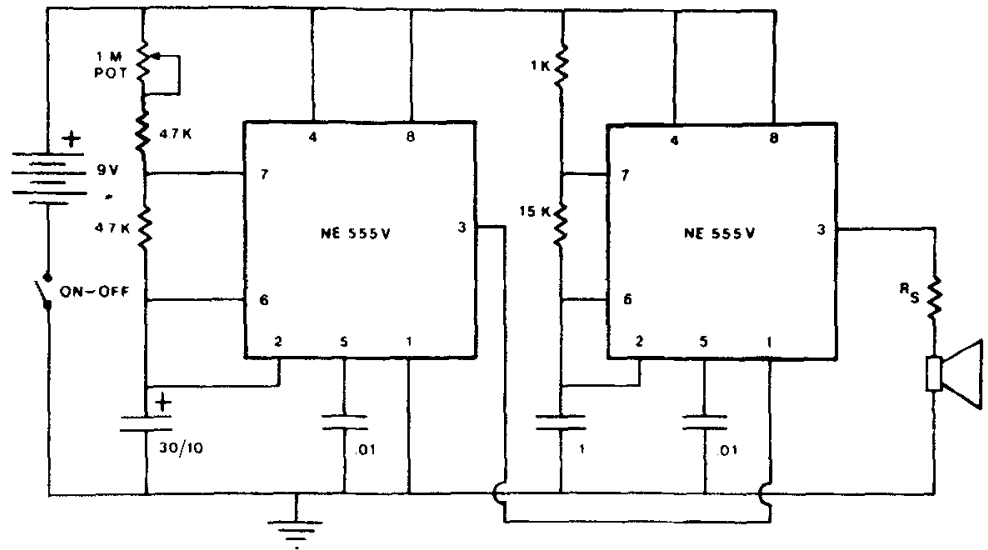




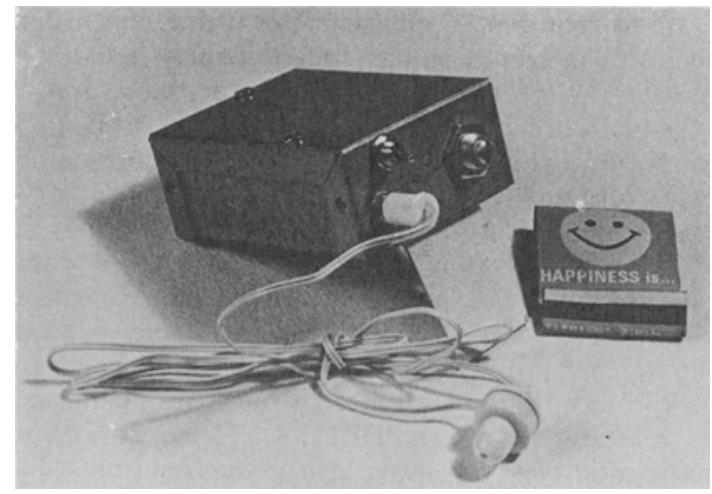

Fig. 2. The assembled unit, illustrating its smallness.

clip, one Arrow-Hart CT5-3-P single-pole double-throw subminiature toggle switch. Mounted on a circuit board, the assembled unit can fit into an LMB No. 772 tite-fit chassis box.

Many laboratories usually have some of the components routinely available, while other components can be obtained inexpensively from local electronics dealers. The Signetic integrated timer circuits are $\$ 1.10$ individually, or $\$ 0.90$ each when purchased in quantities of 25 or more. The potentiometer is about $\$ 1.25$ and the subminiature toggle switch about $\$ 1.30$. All other components are relatively inexpensive, and each unit can be constructed for approximately $\$ 8$. Most components are available at the usual electronics outlets. The timer circuits can be obtained from the Signetics Corp., 811 East Arques Avenue, Sunnyvale, California 94086.

Because the basic timing circuit utilizes small integrated circuits, the assembled instrument is quite small. It is $80 \times 52 \times 28 \mathrm{~mm}$ in size and completely assembled weighs $135-140 \mathrm{~g}$. The assembled unit is shown in Fig. 2.

In our laboratory, these units are used for both behavioral research and instructional purposes. Much of our research uses time-sampling observational procedures, and our laboratory course in animal behavior includes a considerable emphasis on acquiring systematic and rigorous observational skills. The present device is by far the most effective signal generator we have used in either setting.

(Received for publication October 23, 1972; accepted November 5, 1972.) 